\title{
Letter to the Editor in response to the article by Borg et al
}

Dear Editor,

As Australian First Nations people, public health practitioners and academics, we would like to thank Borg et al. for the raising the importance of increasing influenza vaccination rates [1]. The coverage of influenza immunisation in Australian First Nations children is close to $50 \%$ in the Northern Territory but is very low in most other states, thus this is a topic worthy of research. We would, however, like to offer an additional interpretation of their study results.

Raising immunisation coverage from $4 \%$ to $6 \%$ is reported in the paper as a statistically significant result. We feel the most important result is that at least $94 \%$ of children remained unimmunised.

Whilst the authors acknowledge that the issue requires further research, we would like to offer an alternative perspective on strategies to increase influenza vaccination rates in First Nations communities. For example, instead of focusing on letters and pamphlets for parents and guardians as modes of communication, as described in the article, we suggest that focusing on Cultural Governance is most important. First Nations people must have a real say about defining the issue/s, suggesting the solutions to be trialled and interpreting results.

It is not clear to what degree the study reported in Borg et al. involved First Nations people in study design and governance or the intervention implementation. We strongly advocate exploring models of vaccine delivery that are flexible, family-centred, and characterised by beneficial robust partnerships [2]. We recommend establishing a culturally appropriate governance structure at the outset of work undertaken in this area, whereby First Nations people make a real contribution and participate fully in shared decision-making. If we continue to conduct studies that are not led by First Nations people, then they are likely to yield the same results, perhaps with little to no benefit to the health of First Nations people. We believe First Nations people can be centrally positioned in this type of work, in spaces where there is shared power and empowerment, to ameliorate the health and social impacts of colonisation. 
Thus we propose an approach that is informed, developed, led and governed by Australian First Nations peoples [3]. Genuine, meaningful and respectful engagement and action that facilitates active and equal participation is critical in the development, implementation, interpretation of findings, and dissemination of any health program or research [4].

\section{Acknowledgements}

We would like to acknowledge and thank Patrick Cashman, Ross Andrews, Peter Massey, and David Durrheim for their continued support and being willing to give up space and share power ensuring we as First Nations people have a voice.

Katrina Clark, National Centre for Immunisation Research \& Surveillance, Westmead, New South Wales, Australia

Natalie Allan, Hunter New England Local Health District, Population Health, Tamworth, New South Wales, Australia

Paula Taylor, Hunter New England Local Health District, Population Health, Wallsend, New South Wales, Australia

Adrian Miller, Office of Indigenous Engagement, Central Queensland University, Townsville, Queensland, Australia

Sandra Campbell, Indigenous Health, Research Division, Central Queensland University, Cairns, Queensland, Australia

\section{References}

Borg K, Sutton K, Beasley M, et al. Communication-based interventions for increasing influenza vaccination rates among Aboriginal children: A randomised controlled trial. Vaccine 2018;36(45):6590-795.

Thomas S, Cashman P, Islam F, et al. Tailoring immunisation service delivery in a disadvantaged community in Australia; views of health providers and parents. Vaccine 2018;36(19):2596-603.

Jamieson LM, Paradies YC, Eades S, et al. Ten principles relevant to health research among Indigenous Australian populations. Med J Aust 2012;197(1):16.

National health and medical research council (Australia). Values and ethics: Guidelines for ethical conduct 